\title{
CONSERVACIÓN DE INSECTOS EN LA AMAZONIA
}

\section{INSECT CONSERVATION IN THE AMAZON}

\author{
Marc J. Dourojeanni ${ }^{1}$
}

\begin{abstract}
Resumen
La porción del bioma amazónico incluida en áreas protegidas y territorios indígenas se ha duplicado durante las últimas tres décadas. Hoy cubre el $51 \%$ de la Amazonia. Los insectos a menudo se consideraron en los estudios previos. Sin embargo, la mayoría de las especies de invertebrados en áreas protegidas de la Amazonía son desconocidas. La situación de las especies en la parte no protegida empeora a medida que se expande la agricultura, la minería y la tala selectiva, especialmente impactando en especies endémicas en valles aislados en la porción andino-amazónica y en la periferia de la Amazonía. Además, las consecuencias del efecto de borde empeoran a medida que se multiplican los fragmentos forestales. El cambio climático tendrá graves consecuencias. La contaminación del agua tiene un impacto creciente poco medido en los invertebrados acuáticos. La taxonomía, especialmente en los países amazónicos, sigue sin ser atractiva para los entomólogos jóvenes o para las, cada día más pobres, instituciones científicas. Así, el ya difícil tema de la sistemática está empeorando a pesar de nuevas tendencias, como los códigos de barras de ADN. La preocupación por la biopiratería aumenta los obstáculos que los gobiernos imponen para la investigación científica. A pesar de todo, el balance general para la conservación de insectos es más positivo que negativo, ya que se puede asumir que la entomofauna está tan protegida en áreas protegidas como cualquier otro animal o planta. Se discuten algunas alternativas para mejorar la conservación de insectos en la región.
\end{abstract}

Palabras clave: insectos, Amazonia, situación, conservación, áreas protegidas.

\begin{abstract}
During the past three decades the portion of the Amazon biome included in protected areas and indigenous territories has more than doubled. Today it covers $51 \%$ of the Amazon. New protected areas are biologically more representative than in the past thanks to improved scientific tools such as rapid biological assessments and prioritization based on systematization of records. Insects were often considered in these studies. However, not all protected areas have equal value for conservation. Also, most invertebrate species in protected areas as in the Amazon are still unknown. The situation in the still forested non-protected Amazon is worsening. Agriculture and mining are rapidly growing as well as selective logging, especially impacting in endemic insect species in isolated valleys in the Andes-Amazon portion and in general, in Amazon's periphery. Additionally, the consequences of border effects are worsening as forest fragments multiply. Climate change will have an enormous impact. Water pollution is a growing but little measured impact in aquatic invertebrates. Taxonomy, especially in the Amazon countries, continues not being attractive to young entomologist nor to scientific institutions. The whole issue of systematics is worsening despite new trends such as DNA bar codes. Biopiracy susceptibilities are increasing obstacles for research. The overall balance for insect conservation is still more positive than negative as it can be assumed that the entomofauna is as protected as any other animal or plant. A few alternatives to improve insect conservation in the region are discussed.
\end{abstract}

Key words: insects, Amazon, status, conservation issues, protected areas.

\section{Introducción}

El interés por la conservación de insectos, es decir, mantener poblaciones representativas y viables de sus especies, es tan antiguo como lo ha sido para la mayoría de las otras formas de vida. Sin embargo, la estrategia de establecimiento de áreas protegidas, que ya tiene un siglo y medio y que ha sido la única que ha sido eficiente para conservar la biodiversidad, ignoró en gran medida los insectos. Estas áreas se reservan principalmente en función de su representatividad ecológica y de la presencia confirmada de plantas y animales originales, especialmente de los que consideran raros o en peligro de extinción. Es solo excepcionalmente que los insectos, como otros invertebrados, fueron objeto de consideración especial, en parte debido a la falta de información entomológica y, sobre todo, porque se asume que estas especies más pequeñas están protegidas de facto en parques y 
reservas $o$ en cualquier muestra de ecosistema relativamente intacta.

Wilson (1987) ha subrayado dramáticamente la necesidad de un enfoque más serio para la conservación de insectos. Varias publicaciones hicieron contribuciones importantes al respecto e iniciaron la búsqueda de formas prácticas de mejorar la conservación in situ (Collins \& Thomas, 1991; Samways, 2005; Cardoso et al., 2011). El número de artículos dedicados a este tema ha crecido drásticamente en décadas recientes. Desde 1997, Springer patrocina una revista internacional totalmente dedicada a la conservación de insectos e invertebrados. Esta revista ya ha acumulado más de 1500 artículos. Las recientes alarmas sobre la disminución de las poblaciones de insectos (Hallmann et al., 2017; Sánchez-Bayo \& Wychuys, 2019; Le Page, 2019) están agregando urgencia a este tema. Como también recordó el IPBES (2019), la tasa global de extinción de especies ya es por lo menos desde varias decenas a cientos de veces mayor que la tasa promedio en los últimos 10 millones de años y se está acelerando. Reconocen que la proporción de especies de insectos en peligro de extinción es incierta. Pero, la evidencia disponible respaldaría un estimado tentativo del 10 por ciento. Es decir que de unos 8 millones de especies animales y vegetales existentes (el 75\% de las cuales son insectos), alrededor de 1 millón están en peligro de extinción.

En 1989, el autor presentó una revisión sobre la conservación de insectos en América Latina (Dourojeanni, 1990). Ahora, treinta años después, su intención es evaluar los cambios y las tendencias que se han producido, concentrándose en la situación en el bioma amazónico. Los 7.5 millones de kilómetros cuadrados del bioma amazónico son compartidos por 9 países, de los cuales el dominio de Brasil se extiende sobre más del 68\%, en la cuenca baja del Amazonas. Perú, con $13 \%$, Bolivia con $11 \%$ y Colombia, Ecuador y Venezuela cubren en total otro $27 \%$ del bioma. Estos países andinos son responsables de la parte superior de la cuenca del Amazonas, comenzando en la línea de árboles, a unos 3800 metros de altura.

No se necesitan superlativos para describir la biodiversidad amazónica, incluidos los insectos. Se ha estimado que alrededor de 40000 especies de insectos se encuentran en una sola hectárea de bosque amazónico (National Research Council, 1982). Mientras que toda Europa tiene menos de 500 especies de mariposas, el Parque Nacional Manu en Perú (en estudios sobre apenas unas 4000 hectáreas) tiene 1300 especies. El Parque Nacional Tambopata también en Perú (estudio sobre 5500 hectáreas) tiene al menos 1 231 especies (Butler, 2017). No hay actualizaciones globales para la Amazonía, pero solo para proporcionar algunos números indicativos, Mittermeier et al. (1997) mencionan, en orden, Perú, Brasil, Colombia, Bolivia y Venezuela como los cinco principales países con respecto a la diversidad mundial de mariposas. Estos países destacan por sus endemismos, así como diversidad de Papilioninae y Cicindelinae. La mayoría de las especies descritas provienen de la región amazónica de estos países.

Lewinsohn \& Prado (2005) estimaron la población de insectos identificada de Brasil entre 80750 y 109 250 especies. Más tarde, Rafael et al. (2012) confirmaron 90300 especies de insectos identificadas en Brasil de una diversidad estimada en alrededor de 380000 especies. De ser así, la entomofauna conocida de este país representaría el $24 \%$ de las especies que se supone allí existan. Se puede esperar que el $60 \%$ o más de las especies conocidas y el $90 \%$ o más de las especies aún por conocer ocurran en la Amazonía. Sin embargo, otras especulaciones sitúan mucho más alto el número total de especies en América del Sur, especialmente de la Amazonía (Erwin, 1983a; Lewinsohn \& Prado, 2005; Chapman, 2009).

En el Perú se ha estimado la diversidad de insectos identificada en 41300 especies (Aguilar et al., 1995) y, en Colombia, en 35000 especies (Amat-G. et al., 1999). Estos últimos autores hicieron una cuenta nacional detallada para algunos grupos, como Coleoptera e Hymenoptera. Varios países han producido estadísticas parciales de especies identificadas, a nivel de familia, especialmente para los lepidópteros. No hay duda de que el número real de especies de insectos en la Amazonía de los países andinos, tanto como en Brasil, es de cuatro a cinco veces mayor que el identificado, especialmente con respecto a las especies endémicas.

Es importante notar el número muy pequeño de especies de insectos de América del Sur que se incluyen en la Lista Roja de Especies Amenazadas de la UICN (IUCN, 2018). Solo se citan 8131 especies a nivel mundial y solo 907 para América del Sur, de las cuales casi todas provienen de fuera de la Amazonía. Esto representa solo el $1 \%$ de los insectos descritos para Brasil y el $0.24 \%$ de los que se cree existen allí. Y, para completar la escena, la UICN clasifica el $88 \%$ de las especies amenazadas como "probablemente amenazadas" y "sin datos suficientes", lo que evidencia el bajo nivel de confianza del ejercicio. Solo dos especies se consideran extintas, 16 estarían en peligro crítico, 32 en peligro y 35 serían vulnerables. En Perú, solo 20 invertebrados están incluidos en la lista roja de la fauna nacional y solo dos se consideran en situación crítica (SERFOR, 2018). De otra parte, existiría evidencia de que el criterio de la UICN para medir la población de insectos no es adecuado (Fox et al., 2018).

Siendo la entomofauna de la Amazonía tan poco conocida resulta evidente que la información disponible sobre la distribución, la población y el estado de conservación de la mayor parte de la especie es aún más limitada. Existen excepciones para algunas especies económicamente útiles, como las abejas sin aguijón (Schwarz, 1932; Parra \& González, 2000; Freitas et al., 2009; Prado et al., 2017), así como para 
plagas agrícolas como las hormigas cortadoras de hojas o, obviamente, para los insectos que transmiten enfermedades. Además, hay información para algunos otros grupos de interés especial, como las abejas de orquídeas (Roubik \& Hanson, 2004). Por lo tanto, la esencia de la conservación de los insectos amazónicos, por el momento, debe apelar a los esfuerzos generales para conservar muestras representativas de los ecosistemas amazónicos y su biodiversidad, los insectos se benefician tanto como las plantas y otros animales.

\section{Cambios mayormente positivos}

\section{Áreas protegidas: extensión y categorías}

La mejor noticia para la conservación de insectos en el Amazonas es el aumento considerable de la porción del bioma que ahora se incluye en áreas protegidas que en teoría están manejadas. También es importante mencionar la expansión de las tierras indígenas oficialmente reconocidas. Como se muestra en la Tabla 1, en los últimos 30 años la superficie del bioma amazónico bajo algún tipo de protección legal se ha más que duplicado, pasando de 1.81 millones de kilómetros cuadrados a 4.35 millones de kilómetros cuadrados. Hoy más de la mitad del bioma amazónico está protegido legalmente in situ. Las áreas protegidas generalmente tienen infraestructura y equipos básicos y están guardadas por guardaparques y la mayoría de ellas tienen al menos un profesional residente. Los territorios indígenas son defendidos por sus miembros y reciben apoyo gubernamental cuando es necesario.

Tabla 1. Extensión y porcentaje de la Amazonía cubierta por áreas protegidas y territorios indígenas.

\begin{tabular}{lrrrr}
\hline & 1989 & \multicolumn{2}{c}{2019} \\
& Área $\left(\mathrm{Km}^{2}\right)$ & $\%$ & Área $\left(\mathrm{Km}^{2}\right)$ & $\%$ \\
\hline $\begin{array}{l}\text { Tierra } \\
\text { indígena }\end{array}$ & 1060958 & 13.6 & 2368936 & 28.0 \\
$\begin{array}{l}\text { Áreas } \\
\text { protegidas }\end{array}$ & 751803 & 9.7 & 1984569 & 23.4 \\
$\begin{array}{l}\text { Total } \\
\text { Fuentes : RAISG (2019) y CADMA (1992). }\end{array}$ & & & \\
\end{tabular}

En la Amazonía, las áreas protegidas, así como la mayoría de los territorios indígenas, son muy grandes o grandes, a menudo con más de un millón de hectáreas y generalmente con varios cientos de miles de hectáreas. Las áreas protegidas pertenecen a dos grupos de categorías bien definidos. Las que están estrictamente protegidas y donde, en teoría, no se permite su ocupación por la población ni actividades económicas, excepto el turismo. Éstas se conocen como categorías de "uso indirecto". Este grupo incluye parques nacionales, reservas biológicas, estaciones ecológicas y santuarios nacionales. El otro grupo, conocido como "uso directo" o áreas protegidas "blandas", incluye todas las categorías de áreas protegidas que permiten población dentro y niveles variables de actividades económicas que se supone que sean sostenibles. Este grupo incluye las categorías regionalmente conocidas como reservas nacionales, reservas comunales, bosques nacionales, reservas extractivas, áreas de protección ambiental, etc. (Dourojeanni, 2019).

Obviamente, el nivel de protección efectiva de la naturaleza no es igual en ambos grupos ni, tampoco, entre las diferentes categorías del grupo de "uso directo". A pesar de que todas las categorías incluidas en el grupo de áreas "protegidas blandas" permiten la población residente y la explotación de los recursos, incluso la agricultura y la ganadería, algunas son mucho más restrictivas que otras. En Brasil, donde se encuentra el $54 \%$ de la zona amazónica protegida, el $43 \%$ de ella o sea el $12.3 \%$ de la Amazonía brasileña, pertenece a las categorías de "áreas de protección ambiental" y bosques nacionales. En la primera categoría, las regulaciones sobre el uso de la tierra son prácticamente las mismas que en cualquier otro lugar (Dourojeanni \& Pádua, 2013), incluidas grandes porciones dedicadas a la agricultura y la silvicultura. Y en los bosques nacionales, se autorizan concesiones para la tala. Las evidencias muestran que otra categoría brasileña, conocida como reservas extractivas $-9 \%$ de la Amazonía brasileña protegida- pierde una parte importante de los bosques cada año para actividades agrícolas y ganaderas, así como para la tala, a medida que aumenta la población y sus necesidades (Silva et al., 2017).

Como se muestra en la Tabla 2, la proporción de área protegida por cada grupo de categorías es equivalente. La mejor garantía de una conversación a largo plazo es, obviamente, proporcionada por las categorías más estrictamente protegidas agrupadas como "uso indirecto", que cubren solo el $12 \%$ del bioma amazónico. Sin embargo, a largo plazo, esto no es del todo seguro, ya que algunas áreas protegidas de ambos grupos a menudo son sometidas a procesos de eliminación, reducción y degradación, lo que es un problema que es particularmente importante en Brasil, especialmente a nivel de áreas protegidas estatales (Pack et al., 2015).

Tabla 2. Extensión y porcentaje de áreas protegidas de la Amazonía por categoría de manejo.

\begin{tabular}{lrrrr}
\hline & $\begin{array}{r}1989 \\
\text { Categoría }\end{array}$ & Area $\left(\mathrm{Km}^{2}\right)$ & $\%$ & $\begin{array}{r}2019 \\
\text { Area }\left(\mathrm{km}^{2}\right)\end{array}$ \\
\hline $\begin{array}{l}\text { Uso } \\
\text { indirecto }\end{array}$ & 379679 & 4.9 & 1018290 & 12.0 \\
Uso & & & & \\
directo & 371501 & 4.8 & 941943 & 11.1 \\
Provisional & & & & \\
Uso doble & - & - & 35779 & 4.2 \\
Total & 751803 & 9.7 & 1984569 & 0.2 \\
\hline
\end{tabular}

Fuentes: RAISG (2019) y CADMA (1992).

La situación de conservación en tierras indígenas depende de muchos factores, como el tamaño, la densidad de población y el aislamiento. Algunos 
territorios indígenas muy grandes todavía son refugios seguros para la vida silvestre. Sin embargo, a mediano y largo plazo, esta situación puede cambiar drásticamente. Algunos grupos indígenas abren sus tierras a minería ilegal, tala e incluso a agricultura intensiva y pastoreo. Esta tendencia está creciendo a medida que mejora la educación y el contacto con el resto de la sociedad. Por lo tanto, una vez más, el valor de conservación a largo plazo de estas áreas no es evidente, pero es de esperar que al menos una parte permanezca segura durante varias décadas (Dourojeanni \& Pádua, 2007, 2013).

Además, debe señalarse que existen reservas de biosfera y sitios Ramsar en los humedales, que cubren $993071 \mathrm{~km}^{2}$ o el $12.8 \%$ de la Amazonía (RAISG, 2019). Alrededor del $50 \%$ de esta área también se incluye en áreas protegidas y tierras indígenas. El valor de conservación de las reservas de biosfera es muy limitado ya que incluyen grandes porciones de tierra bajo usos económicos intensivos. Finalmente, la dificultad para establecer nuevas áreas de conservación, especialmente aquellas estrictamente protegidas, ya es enorme y aumentará.

Áreas protegidas: representatividad biológica y entomológica

En general, las áreas protegidas establecidas en la Amazonía son más representativas de la diversidad biológica que en otras regiones de América del Sur. Eso se debe a que en los últimos 30 años se han aplicado metodologías mejoradas para seleccionar los lugares a ser conservados. Esto es, en principio, buenas noticias para la entomofauna.

Es un hecho que, hasta la década de 1950, las áreas protegidas de América del Sur como las de otros continentes, se establecieron con base de justificaciones éticas, estéticas y geopolíticas, lo que resultó en la protección de paisajes hermosos, a menudo lagos y montañas cerca de las fronteras del país, con poca consideración por su valor ecológico. Baldi et al. (2017) consideran que la mayoría de las áreas protegidas del mundo son el resultado de la oportunidad en lugar de la representatividad biológica. Este no ha sido el caso en la Amazonía. En ella las áreas protegidas comenzaron a establecerse tarde, solo en la década de 1960 y especialmente en la década de 1970. Por entonces, la mayor parte de la tierra todavía era del dominio público, lo que reducía las restricciones para establecer áreas protegidas. En efecto, los derechos sobre la tierra son el obstáculo más severo para crear un sistema biológicamente representativo de áreas protegidas. De otra parte, el establecimiento de áreas protegidas en el Amazonas siempre ha sido impulsado por los criterios científicos y, como se mencionó anteriormente, la mayoría de ellos son considerables, proporcionando un amplio refugio contra los efectos de borde.

Todas las áreas protegidas de la Amazonía brasileña establecidas hasta fines de la década de 1980
(Wetterberg et al., 1976; Pádua \& Coimbra, 1979; IBDF, 1982) siguieron la información científica disponible, especialmente basada en la teoría de los refugios del Pleistoceno (Brown, 1972, 1978, 1979, 1982, 1984, 1987a, 1987b; Prance, 1982; Nelson et al., 1990; Morrone, 1994), incluidas las consideraciones sobre los insectos (Brown, 1978, 1979, 1984, 1987a, 1987b, 1988). En los países andinos, las áreas protegidas del Amazonas se planificaron en base a información sobre zonas ecológicas o de vida (Tosi, 1960) y datos de campo sobre especies de mamíferos y aves en peligro de extinción (Grimwood, 1968; Dourojeanni, 1968; 2018a, Dourojeanni \& Ponce, 1978). A partir de la década de 1990, se aplicaron metodologías de justificación más detalladas. Brasil desarrolló detallados esfuerzos conjuntos de especialistas en cada taxón de plantas y animales, incluidos los insectos, para definir zonas prioritarias para el establecimiento de nuevas áreas protegidas (IBAMA, 1991; Kress et al., 1998) o utilizando algunos grupos de insectos, como los escarabajos tigre (Cicindelidae) como indicadores (Pearson, 1992, 1994).

Desde la década de 1990 en los países andinoamazónicos, se hizo un esfuerzo en la aplicación de evaluaciones biológicas rápidas (Longino et al., 2000) que a menudo incluían entomólogos en el equipo. Esto se ha utilizado para prácticamente todas las áreas protegidas creadas desde la década de 1990 (Foster et al., 1994; Schulenberg \& Awbrey, 1997; Vriesendorp, 2006; Pitman et al., 2004). Por supuesto, estos inventarios de insectos nunca eran completos $\mathrm{y}$, a menudo, dependían solamente de los especialistas que formaban parte del equipo. Solo por mencionar un caso, en el estudio para el Parque Nacional Cordillera del Cóndor, entre Ecuador y Perú, dos entomólogos registraron 474 especies de Lepidópteros y 34 Scarabaeidae (Schulenberg \& Aubrey, 1997). Eso es bueno, pero está muy lejos de ser una imagen total que, en cambio, sí se proporcionó para mamíferos, aves y reptiles y, en gran medida, para especies de árboles. En otros casos, la justificación se ha basado en extensas referencias científicas acumuladas durante décadas (Tovar et al., 2009). Hay casos en que la ciencia advirtió sobre situaciones específicas que necesitaban atención. En Perú, este ha sido el caso de los ecosistemas de arena blanca (Garcia-Villacorta et al., 2016) que son ricos en endemismos.

A pesar de la considerable extensión del bioma amazónico ya protegido, varios autores señalan deficiencias en la cobertura de algunos grupos de animales y plantas, incluidos los insectos. Oliveira et al. (2017a, 2017b) llevaron a cabo un análisis de lagunas con respecto a los vertebrados, artrópodos y angiospermas usando datos filogénicos en la red brasileña de áreas protegidas. Los resultados muestran que el conocimiento de la biodiversidad en las áreas protegidas sigue siendo escaso, ya que el $71 \%$ de ellas 
tiene menos de 0.01 registros de especies por $\mathrm{km}^{2}$. Casi el $55 \%$ de las especies y aproximadamente el $40 \%$ de los linajes evolutivos no se encontraron en las áreas protegidas, mientras que la mayoría de las especies tienen menos del $30 \%$ de su distribución geográfica dentro de ellas. El sistema actual de áreas protegidas no protegería a la mayoría de las especies endémicas. Otra investigación, en Perú, llegó a una conclusión similar (Fajardo et al., 2014). Sin embargo, en ambos estudios, la mayoría de los resultados se refieren más a áreas protegidas fuera que dentro de la Amazonía.

Es obvio que la mayor parte de los insectos y otros invertebrados que existen en las áreas protegidas del Amazonas son parte de una caja negra. Sin embargo, es importante resaltar que gran parte de la investigación entomológica en la Amazonía se ha llevado y se está llevando a cabo cada vez más en áreas protegidas (Brown, 1984; Pearson, 1985; Paulson, 1985; Lamas, 1985, 1979; Fisher, 1985; Wilkerson \& Fairchild, 1985; Dressler, 1985; Erwin, 1985, 1991a, 1991b; Emmel \& Austin, 1990; Robbins et al., 1997; Vicente et al., 2016). Obviamente, esto es ventajoso para los investigadores, que encuentran un lugar seguro y apoyo para trabajar y para el parque o la reserva, que descubren la riqueza protegida (Dourojeanni, 2018b) Cuando se procesa, esta información puede proporcionar indicaciones para otras medidas de conservación.

Conservación ex situ y promoción de entomología

El turismo se ha desarrollado significativamente en la Amazonía y los insectos son parte de las atracciones que se ofrecen a los visitantes. Especialmente en Brasil, Colombia, Perú y Ecuador, en las últimas dos décadas se estableció un número creciente de mariposarios o zoológicos. Brasil es, con mucho, el país con más instalaciones de este tipo, algunas son públicas, como las administradas por la Fundação Zoo-Botánica y Fiocruz, pero la mayoría son privadas, como Aguias da Serra, Flores que Voam, Mangal das Garças, SESC Pantanal, Serelepe, etc. En Colombia también hay varios jardines de mariposas, como Quindio, Paway y Andoke. En Perú, entre otros, vale la pena mencionar Tambopata, Machu Picchu y Pilpintwasi. En Ecuador los más conocidos son Mindo, Cotococha, Machay y La Carolina. La mayoría trabaja solo con mariposas, pero algunas tienen otros insectos. El número de especies generalmente se limita a unos pocos cientos o menos, sin embargo, algunos conservan especies raras o valiosas.

Otro desarrollo es la oferta de visitas entomológicas y cursos de campo o capacitación, a menudo en reservas naturales privadas $o$ estaciones de investigación en la selva. El turismo entomológico es ofrecido tanto comercialmente como por organizaciones no gubernamentales en varios países, como en el caso de la Lepidoptery Butterfly Tours en la Amazonía del Ecuador. Se ofrece un curso bastante conocido en la Estación de Campo Las Piedras en Perú, operado por la Alianza para una Amazonía Sostenible.

Es evidente que estas iniciativas no son una respuesta a las necesidades de conservación de la entomofauna, pero, como su número y calidad aumentan se convierten en una fuente de nuevas vocaciones entomológicas muy necesarias.

\section{Cambios negativos}

Deforestación, fragmentación forestal, degradación forestal

La deforestación significa la eliminación absoluta de toda la biota existente por encima y, en gran medida, también por debajo del suelo. El proceso comienza con la extracción de árboles valiosos y continúa con la eliminación y quema de toda la vegetación. Para los insectos es el final, pero puede estar precedido por una explosión de población de insectos de descomposición de la madera y de la materia orgánica, que encuentran una acumulación inusual de lugares para la oviposición. $\mathrm{Su}$ ciclo de vida generalmente se ve interrumpido por la quema. En fincas más grandes, la madera muchas veces no se quema, sino que se desmenuza y se acumula, pero la distancia al bosque restante es demasiado grande para que muchas especies alcancen ese material.

Se estima que del 18 al 20\% del bioma amazónico ha sido desprovisto de sus bosques originales (Lovejoy $\&$ Nobre, 2018). Brasil responde por el $76 \%$ del área deforestada, seguido por Bolivia (10\%) y Perú (7\%). En 1989, el área deforestada en la Amazonía brasileña cubría $419010 \mathrm{~km}^{2}$ y su bosque restante todavía ocupaba el $90.4 \%$. En 2018, la deforestación ya cubría $792051 \mathrm{~km}^{2}$ y la porción restante de la Amazonía brasileña se ha reducido al $80.7 \%$ (INPE, 2019). En Perú, la deforestación sigue una tendencia similar, ya que solo entre 2001 y 2018 se desnudaron alrededor de 250000 km² $^{2}$ (Finer \& Mamani, 2019). La deforestación acumulada en el Perú probablemente alcanza cerca del 18\% (Dourojeanni, 2011).

Más del $90 \%$ de la deforestación ocurre fuera del espacio cubierto por áreas protegidas y territorios indígenas (51\% de la Amazonía), lo que significa que la porción de bosques no protegidos aún en pie cubre alrededor del 30\% del bioma (Dourojeanni, 2019). Cabe señalar que alrededor del $20 \%$ del área previamente deforestada está cubierta por diferentes etapas de bosques secundarios que ocupan tierras de cultivo abandonadas. El valor biológico de estos bosques secundarios, en términos de biodiversidad, es mucho menor que los bosques originales (Gibson et al., 2011) y su permanencia es solo temporal (Jakovac et al., 2015). Los bosques primarios son insustituibles para mantener la biodiversidad tropical.

Uno de los mayores problemas con respecto a la deforestación en curso es que ocurre principalmente en las periferias de la Amazonía, especialmente en su porción sur y sureste en Brasil y en su porción 
occidental en los países de los Andes y la Amazonía. De hecho, la deforestación ha estado y sigue ocurriendo en ecotonos de la Amazonía con ecosistemas vecinos, especialmente el Cerrado y el Pantanal en Brasil (Marques et al., 2019) y los punas, yungas y páramos en los países andinos. Esos ecotonos albergan especies de ambos ecosistemas, pero también pueden tener especies que son únicas para el ecotono. Por lo tanto, los ecotonos también pueden considerarse hábitats de mayor diversidad genética y servir como puentes entre las poblaciones. Excepto cuando se establecieron áreas protegidas en parte de estos ecotonos, la mayoría ya se han perdido debido a la deforestación. Las especies allí perdidas posiblemente nunca se conozcan.

El endemismo a menudo resulta del aislamiento a largo plazo. Esta es una situación bastante común en la parte superior de la región andina-amazónica, ya que numerosos valles profundos que descienden de los Andes entre 3800 y alrededor de 600 metros pueden estar bastante aislados por montañas, gargantas y otros obstáculos geográficos. Estas son las condiciones en las que se están registrando la mayoría de las especies endémicas de animales y plantas y este es probablemente también el caso de los insectos. Lamentablemente, una gran parte de estas áreas ya ha sido destruida, consumida por el fuego para abrir y mantener pastizales en laderas muy susceptibles a la erosión y, en altitudes más bajas, por coca y otras plantaciones en laderas escalonadas.

El modelo de ocupación de la tierra amazónica crea una serie de islas de vegetación natural o seminatural cuyo futuro es incierto. Dependiendo de su tamaño, estos fragmentos de bosque pueden mantener durante un tiempo una gran parte de su diversidad biológica original (Lamarre et al., 2016) pero se establece una relación tensa de su biota en los bordes con los usos de la tierra circundante, generalmente cría de ganado o especulaciones agrícolas (Haddad et al., 2015). Estas interacciones se han estudiado desde hace mucho tiempo (Lovejoy et al., 1986; Bierregaard et al., 1992) incluso para los insectos. En el caso de Euglossinae, la tasa de vistas de las abejas disminuyó con el tamaño de los fragmentos, a pesar de que las aberturas que las separan los fragmentos y el bosque continuo tienden a ser de solo unos cien metros. Las especies de abejas atraídas por los olores en el espacio abierto diferían de las del bosque, lo que indica que había poco potencial para la polinización del bosque por parte de las especies de campo abierto (Powell \& Powell, 1987). Otros estudios confirmaron la respuesta de los insectos a la reducción del área forestal, incluyendo abundancias alteradas, riqueza o composición de especies en comparaciones de fragmentos de diferentes tamaños, áreas fragmentadas $\mathrm{y}$ no fragmentadas, $\mathrm{o}$ comparaciones de bordes de bosques e interiores de bosques. Estas respuestas tienden a ser idiosincrásicas, con algunos grupos que muestran disminuciones pronosticadas de la abundancia o diversidad en los fragmentos, mientras que otros no muestran respuesta o incluso aumentan (Vasconcelos \& Bruna, 2012). También se ha informado de la invasión de fragmentos de bosque por especies externas (Martins, 1989). Todos los estudios demuestran que la descomposición biológica de los fragmentos de bosque es inevitable (Laurance \& Vasconcelos, 2009; Laurance et al., 2011).

Especialmente durante las últimas dos décadas, la degradación forestal se ha destacado como otro tema clave para la supervivencia de la biodiversidad (Foley et al., 2007). Obviamente, la información sobre la degradación forestal es mucho menos precisa y difícil de definir y evaluar que la deforestación, pero las estimaciones indican que alrededor del $60 \%$ de los bosques restantes sufrieron algún nivel de degradación. La tala selectiva, la forma más común de explotación forestal en la Amazonía, es la principal causa de la degradación de los bosques (Souza \& Roberts, 2005). Este proceso ocurre durante décadas, ya que la tala selectiva implica olas de extracción exhaustiva de madera que cada vez se dirigen a diferentes especies a menudo en los mismos lugares. Esto es especialmente grave para los insectos que son específicos de las copas de los árboles. Como lo demostró Erwin (1982, 1983a), los insectos del dosel son bastante específicos para cada especie de árbol. También demostró que el 83\% de las especies de escarabajos en las muestras se encontraron solo en las muestras de uno de los tipos de bosque, el $14 \%$ de las especies se compartieron entre dos, y solo el $1 \%$ de las especies de escarabajos se encontró en los cuatro tipos de bosque (Erwin, 1983b). Por lo tanto, la tala selectiva exhaustiva de especies comerciales puede tener un impacto tremendo en los insectos de las copas de árboles, pero poco conocido y escasamente evaluado.

Calentamiento global, contaminación del agua

Obviamente, se espera que los insectos sean susceptibles a los cambios climáticos. Bradford \& García (2018) consideran que el cambio climático ha sido el principal impulsor de la reducción de la población de insectos en una investigación a largo plazo realizada en un bosque tropical lluvioso de Puerto Rico. Descubrieron que la biomasa de peso seco de los artrópodos capturados en muestras de barrido había disminuido de 4 a 8 veces, y de 30 a 60 veces en trampas adhesivas entre 1970 y 2012 , mientras que la temperatura aumentó $2{ }^{\circ} \mathrm{C}$. Otras investigaciones también atribuyen especial importancia al cambio climático para la disminución de la población de insectos (Liebhold \& Bentz, 2011; Lister \& García, 2018) pero, obviamente, dicho cambio también aumentará las poblaciones de algunos de ellos. En la Amazonía, se espera que el impacto del cambio climático combinado con la deforestación sea muy grave (Malhi et al., 2008). El análisis del impacto del calentamiento global en miles de especies arbóreas encontró que los efectos del cambio climático están 
alterando la composición de las especies arbóreas de la selva, pero no lo suficientemente rápido como para mantenerse a la par con el entorno cambiante (Esquivel-Muelbert et al., 2018), lo que significa muy malas noticias para las especies de artrópodos asociadas a ellos. El impacto debería ser máximo en la región andino-amazónica, donde los estratos altitudinales brindan incluso menos oportunidades de adaptarse a los cambios. Otra consecuencia del calentamiento climático, combinado con la deforestación y la fragmentación y degradación de los bosques, es el aumento de los incendios forestales en áreas que alguna vez fueron a prueba de fuego (Aragão \& Simabukuru, 2010). Todos los incendios forestales son perjudiciales para los insectos, pero algunos tipos son peores que otros.

Otra mala noticia para los insectos en el Amazonas es la creciente gravedad e irregularidad de la secuencia de inundaciones y sequías (Barichivich et al., 2018). La causa es generalmente atribuida a la deforestación en lugares más altos. Sus implicaciones para la biota son muy complejas, afectando directamente a las poblaciones de insectos ubicadas en la vegetación ribereña y en las várzeas a lo largo del sistema fluvial del Amazonas. En efecto, estos cambios son abruptos, extremos y a menudo fuera de la estación prevista. En sus períodos secos pueden eliminar parte de la vegetación.

Los insectos acuáticos están seriamente influenciados por los cambios en el uso del suelo y la integridad del hábitat. Sánchez-Bayo \& Wyckhuys (2019) señalan que cuatro taxones acuáticos principales (Odonata, Plecoptera, Trichoptera y Ephemeroptera) ya han perdido una proporción considerable de especies. Nessimian et al. (2008) destacaron la importancia de la vegetación ribereña en el mantenimiento de la biota de los arroyos y su función como corredores ecológicos, y confirmaron el hecho de que los riachos rodeados de pastos tienen una diversidad reducida de población e insectos. Sin embargo, el problema principal para los invertebrados acuáticos en el Amazonas es la contaminación del agua (Castello et al., 2012). La agricultura de labranza en limpio, a menudo en laderas empinadas, así como la ganadería mal manejada y la minería a campo abierto están produciendo enormes volúmenes de sedimentos. Además, el uso de agroquímicos es intenso y la minería ilegal está difundiendo mercurio, así como otros productos químicos, en toda la región. La explotación petrolera en Ecuador, Perú y Bolivia libera enormes volúmenes de petróleo perdido $\mathrm{y}$, hasta hace una década, todas producían aguas residuales. Además, la población humana ya llegó a unos 40 millones de personas y los desechos urbanos e industriales se vierten sin tratamiento en los ríos (Fidelis et al., 2008; Couceiro et al., 2007)). En los numerosos arroyos cuyas ofertas de agua han sido modificadas por la construcción de carreteras o están cerca de comunidades, el grado de sedimentación es muy intenso y la fauna acuática se reduce a muy pocas especies.

Investigación entomológica, taxonomía

La investigación entomológica ha tenido un aumento moderado en los países amazónicos en las últimas tres décadas. Esto parece ser el resultado del crecimiento vegetativo del número de investigadores más universidades, más estudiantes- ya que la situación general de la investigación es probablemente proporcionalmente peor hoy que a fines de la década de 1990, debido a las crecientes deficiencias presupuestales de las instituciones académicas y científicas sudamericanas. Recolectar y especialmente preservar colecciones es muy costoso. Por ejemplo, el gobierno del presidente Bolsonaro anunció a principios de 2019 una reducción significativa de los ya escasos presupuestos de las universidades públicas brasileñas. Accidentes como la destrucción por incendio del centenario Museo Nacional de Brasil en Río de Janeiro, que poseía la mayor y mejor colección de insectos del país, se atribuye en gran medida a restricciones presupuestarias.

La financiación de fuentes internacionales $\mathrm{y}$ especialmente de instituciones nacionales no gubernamentales no suele destinarse a la investigación entomológica. Por ejemplo, la Fundación del Grupo Boticario, la organización de financiación privada de conservación mayor de Brasil, financió 1581 proyectos de conservación e investigación entre 1991 y 2019, peros solo 70 se ocuparon de las relaciones de los insectos con plantas $\mathrm{u}$ otros grupos de animales, a menudo con insectos útiles como las abejas nativas. Sin embargo, uno sólo de sus proyectos, que estudió insectos acuáticos en la Amazonía, identificó 24 especies nuevas de Trichoptera (Boticario, 2015).

La mayoría de los entomólogos sudamericanos continúan practicando entomología aplicada (agricultura, salud) o realizan investigaciones en aspectos biológicos. No hay signos de un aumento significativo en el número de taxonomistas latinoamericanos desde que este problema se discutió hace treinta años (Dourojeanni, 1990). El número de taxonomistas en la región apenas asciende a unos cientos de especialistas (Rafael et al., 2012; Amat-G. et al., 1999) a menudo se concentraron en los lepidópteros y algunas familias de coleópteros e himenópteros. Barros de Carvalho (1998) declaró que los taxonomistas de insectos en Brasil son una "especie en extinción". El sesgo taxonómico en la investigación de conservación es un tema bien descrito (Clark \& May, 2002). De 1999 a 2006, la Revista Peruana de Entomología registró 20 nuevas especies de artrópodos, pero solo una no era una mariposa. De todos modos, los científicos brasileños, con el $15-16 \%$ de todos los artículos publicados, son los principales contribuyentes a Zootaxa (un promedio de 32000 páginas / año dedicadas a la sistemática) y son muy activos en varias otras revistas científicas nacionales e internacionales. 
Pero en los países andinos, el número de taxonomistas locales no aumentó en las últimas décadas (Aguilar et al., 1995; Amat-G. et al., 1999) y la mayor parte de la investigación taxonómica es realizada por o con la asistencia de entomólogos extranjeros. Tampoco hay evidencia clara de mejora de la capacidad de identificación de las instituciones científicas del mundo desarrollado que los entomólogos sudamericanos puedan solicitar.

Para agregar dificultades a las descritas, debe mencionarse que la histeria parcialmente justificada relacionada con la biopiratería (Homma, 2005), especialmente pero no solo en Brasil, está dificultando aún más el trabajo de los taxonomistas. Los procedimientos para obtener la autorización para recolectar insectos son muy complejos y largos y, finalmente, pueden enfrentar la ignorancia de un oficial de policía o de aduana, con graves consecuencias para el investigador $\mathrm{y}$ especialmente para el material recolectado. Millones de hectáreas que contienen miles de millones de insectos se queman cada año, pero recolectar insectos para la ciencia o para el pasatiempo es controlado y sanciona do severamente.

A menudo se considera la taxonomía como el principal cuello de botella para mejorar efectivamente la conservación de insectos en la Amazonía. Por lo tanto, se ha depositado la esperanza en nuevas opciones de identificación de insectos que parecen ser más rápidas que la descripción morfológica clásica. El código de barras de ADN se ha convertido en un estándar rentable para la identificación rápida y más precisa de especies (Hebert et al., 2003; Miller, 2007). La sistemática molecular ha llevado a un aumento en las especies recién descubiertas a medida que las diferencias genéticas se hacen más evidentes. Tiene el potencial de acelerar el descubrimiento de nuevas especies, mejorar la calidad de la información taxonómica y hacer que esta información esté fácilmente disponible para los no taxonomistas e investigadores fuera de los principales centros de recolección (Agnarsson \& Kuntner, 2007). Sin embargo, la utilidad práctica de esta alternativa ha sido seriamente criticada (Audisio, 2017). Yang et al. (2015) hicieron otra sugerencia utilizando un sistema automatizado para identificar insectos basado en los contornos de las alas, que parece prometedor para varias órdenes de insectos. Por el momento, ninguna de estas nuevas opciones ha hecho un progreso notable en la taxonomía de insectos de América del Sur. Incluso si las promesas de una identificación más rápida de los insectos se materializan, siempre estarán precedidas por los pasos de recolección, preparación del material, transporte y almacenamiento en colecciones que, bajo la realidad de Amazon, no es tarea fácil.

\section{Opciones para el futuro}

La taxonomía es importante, pero puede no serlo tanto para conservar los insectos en la Amazonía. Sería ideal conocer el estado y la distribución de un alto porcentaje de especies correctamente identificadas para decidir sobre el establecimiento de áreas protegidas. Sin embargo, esto no se hace realmente, incluso con respecto a las especies de árboles, que son incomparablemente mejor conocidas que los insectos. Y, para obtener dicha información sobre los insectos, se requerirá mucho más tiempo que el restante disponible para establecer áreas protegidas. Los insectos son demasiado numerosos para ser tratados como mamíferos o aves. La conservación de los insectos debe considerar soluciones a granel. Y, hasta ahora, el proceso seguido para establecer áreas protegidas en el Amazonas es bastante adecuado para conservar muestras representativas y viables de insectos. De lo contrario, el entomólogo debe luchar por la representatividad ecológica y la efectividad de la gestión mejorada de las áreas protegidas tanto como todos los demás biólogos y ambientalistas. Los entomólogos también deben luchar por cada elemento que ya está en la larga lista de aquellos que tienen la intención de conservar el Amazonas y restringir el desarrollo como de costumbre a no más de lo que es actualmente y hacerlo más sostenible. Esta es la mejor forma disponible actualmente para conservar la riqueza de insectos amazónicos.

Los esfuerzos de los taxonomistas de insectos deben centrarse en los ecosistemas amazónicos que están en riesgo inminente. Estos son, como se mencionó, a lo largo de la frontera de deforestación en todas partes y en los valles de la porción andino-amazónica. Es esencial concentrar los esfuerzos donde el endemismo es más probable. Además, especialmente en especies de árboles comerciales, concentrándose en sus copas, que fueron, son o serán sobreexplotadas. Para compensar la escasez de entomólogos profesionales, es importante empoderar a los entomólogos aficionados que en muchas regiones del mundo hicieron contribuciones considerables a la taxonomía (Gibbs, 1978; Audisio, 2017). En los países amazónicos, este es un territorio inexplorado, ya que, en lugar de promoverlos, las leyes existentes crean todo tipo de obstáculos. Pero esto puede ser cambiado. Brown (1997) revela que el monitoreo de varios grupos de indicadores de insectos por parte de los residentes en una reserva extractiva de Amazon ha ayudado a sugerir pautas para la zonificación sostenible y los regímenes de uso.

La información sobre los insectos en la Amazonía es tan escasa que no es posible implicar que sus poblaciones estén sufriendo una disminución de la biomasa como se indica en otros biomas y situaciones (Hallmann et al., 2017). Sin embargo, este puede ser el caso de ecosistemas específicos en la frontera de la deforestación, nuevamente especialmente en los valles andino-amazónicos, donde el impacto del uso intensivo de pesticidas en las plantaciones vecinas, entre otras intervenciones, como incendios, puede afectar la biota natural restante. 
Con respecto a las amenazas del calentamiento global, es evidente que es de gran interés para la conservación de insectos la promoción del concepto de corredores ecológicos o biológicos, que vincula las áreas protegidas y que permiten el flujo genético y las posibilidades de "escape" para las poblaciones afectadas por la temperatura y los cambios asociados (Rosenberg et al., 1997; Feeley \& Rehm, 2012). Se propusieron varios corredores importantes en la Amazonía, algunos son nacionales y otros son más ambiciosos y vinculan áreas protegidas de países a través de las fronteras. Sin embargo, esta es una empresa muy difícil, ya que depende del tipo de desarrollo que se interponga en el camino y en las políticas nacionales. La mayoría son solo proyectos.

Los corredores biológicos a menudo ponen esperanza en las reservas naturales privadas. Esta es una estrategia de conservación que está creciendo en los países amazónicos, especialmente en Brasil y Perú. Estas reservas y opciones similares como las concesiones de conservación y ecoturismo (Perú) pueden tener desde unas pocas hectáreas hasta unos pocos cientos de miles de hectáreas. Ya cubren un área importante. Estos y otros lugares similares pueden convertirse en una excelente alternativa para conservar especies de insectos que son raras o en peligro de extinción. Pero esto solo se puede hacer en regiones donde la diversidad de insectos es bien conocida y cuando se identifican brechas, como en España (Hernández-Manrique et al., 2012), Escocia (ISI, 2009) o Sudáfrica (McGeoch et al., 2011) y, posiblemente, en el sur y sureste de Brasil (Miranda et al., 2019). En el caso de España, los investigadores encontraron que el $77 \%$ de 52 artrópodos y 27 especies de moluscos que están en peligro o en peligro crítico no estaban representados en el $94 \%$ de las unidades del sistema actual de áreas protegidas. Es verdad que las áreas protegidas españolas son probablemente menos ecológicamente representativas que las del sistema de la Amazonía, pero este resultado muestra la enorme dificultad de conservar insectos, incluso cuando la taxonomía no es el problema principal.

Cuando la destrucción de la naturaleza es tan intensa que no se pueden encontrar áreas naturales para preservarlas, al menos algunas especies pueden sobrevivir en la vegetación ribereña de paisajes agrícolas que algunos países, como Brasil, protegen bastante bien. En los paisajes rurales, la presencia de árboles y arbustos en las configuraciones agroforestales sirve como hábitat para los insectos nativos, incluidos polinizadores e insectos beneficiosos. $\mathrm{La}$ agrosilvicultura, común en la Amazonía, puede reducir el riesgo de exposición de los polinizadores al pesticida (Perry et al., 2016). El uso de pesticidas es generalmente bajo en agroforestería debido a la menor incidencia de plagas y enfermedades. Por ejemplo, Brandle et al. (2004) observaron una mayor densidad y diversidad de poblaciones de insectos en rompevientos que proporcionaban microhábitats variados y una variedad de huéspedes, presas, polen y fuentes de néctar. Brown \& Freitas (2002) incluso encontraron que la conservación efectiva de las comunidades de mariposas se puede lograr en ciudades tropicales mediante el mantenimiento de corredores verdes arbóreos a lo largo de avenidas, calles y cursos de agua entre áreas húmedas moderadamente grandes, no cerca de los centros urbanos más contaminados o contaminados y la inclusión dentro de estas áreas de estanques o arroyos, bosques nativos diversificados y vegetación abierta que incluye abundantes flores ricas en néctar Una experiencia reciente en Sri Lanka confirma la validez de este enfoque (Handunnetti, 2019).

Los últimos 30 años muestran mejoras, nuevas amenazas y nuevas realidades sobre la conservación de insectos en la Amazonía. Evidentemente, el principal hecho positivo es el enorme aumento de un sistema de áreas protegidas bastante ecológicamente representativo que, incluso teniendo en cuenta que las áreas protegidas blandas y los territorios indígenas no ofrecen una garantía total de conservación, está proporcionando la conservación a medio y largo plazo de grandes poblaciones de insectos. Las amenazas continúan para los insectos como para cualquier otra especie. Pero las poblaciones de insectos pueden sobrevivir en áreas relativamente pequeñas, mientras que los mamíferos como el icónico jaguar necesitan áreas muy grandes. Por lo tanto, si bien el conocimiento es útil en todas partes, se le debe dar mayor prioridad a la investigación taxonómica fuera de las áreas protegidas para proporcionar indicaciones para establecer áreas protegidas más pequeñas que aborden especies de insectos en peligro de extinción.

Finalmente, se debe hacer mucho más para incentivar el interés en los insectos en la sociedad en general (Simaika \& Samways, 2018) y especialmente en los jóvenes. Se está dando un paso importante con los jardines de mariposas y similares que se están extendiendo en los países amazónicos, pero las regulaciones deberían modificarse para promover este tipo de iniciativas.

\section{Literatura citada}

Agnarsson I. \& Kuntner M. 2007. Taxonomy in a Changing World: Seeking Solutions for a Science in Crisis. Society of Systematic Biologists. Systematic Biology, 56(3): 531539. DOI: $10.1080 / 10635150701424546$.

Aguilar P., Raven K., Lamas G. \& Redolfi I. 1995. Síntesis de los hexápodos conocidos en el Perú. Rev. Per. Ent., 37: $1-9$.

Amat-G. G., Andrade-C. M.G. \& Fernández F. (Editores). 1999. Insectos de Colombia. Bogotá, Academia Colombiana de Ciencias Exactas, Físicas y Naturales.

Aragão L.E.O.C. \& Shimabukuro Y.E. 2010. The Incidence of Fire in Amazonian Forests with Implications for REDD. Science, 328(5983): 1275-1278. DOI: $\underline{10.1126 / \text { science. } 1186925 .}$. 
Audisio P. 2017. Insect taxonomy, biodiversity research and the new taxonomic impediments. Fragmenta entomologica, 49 (2): 121-124.

Baldi G., Teixeira M., Martin O.A., Grau H.R. \& Jobbágy E.G. 2017. Opportunities drive the global distribution of protected areas. PeerJ - the Journal of Life and Environmental Sciences: Biodiversity and Conservation section, 5: e2989. https://peerj.com/articles/2989/. DOI: 10.7717/peerj.2989.

Barichivich J., Gloor E., Peylin P., Brienen R.J.W., Schöngart J.,. Espinoza J.C \& Pattnayak K.C. 2018. Recent intensification of Amazon flooding extremes driven by strengthened Walker circulation. Science Advances, 4(9): eaat8785. DOI: $10.1126 /$ sciadv.aat8785.

Barros de Carvalho C.J. 1998. Taxonomista de insetos: Uma espécie em extinção no Brasil. Soc. Ent. do Brasil, 23(1): $1-5$.

Bierregaard Jr. R.O., Lovejoy T.E., Kapos V., Dos Santos A.A. \& Hutchings R.W. 1992. The Biological Dynamics of Tropical Rainforest Fragments. BioScience 42(11): 859-866. DOI: $\quad 10.2307 / 1312085$. https://www.jstor.org/stable/1312085.

Boticario (Boticario Group Foundation). 2015. New species of aquatic insects are discovered in the Amazon. Boticario, News of 11 May 2015. http://www.fundacaogrupoboticario.org.br/en/news/page s/new-species-of-aquatic-insects-are-discovered-in-theamazon.aspx.

Bradford C.L. \& Garcia A. 2018. Climate-driven declines in arthropod abundance restructure a rainforest food web. PNAS (Proceedings of the National Academy of Sciences USA), 115(44): E10397-E10406. DOI: https://doi.org/10.1073/pnas.1722477115.

Brandle J.R., Hodges L. \& Zhou X. 2004. Windbreaks in sustainable agriculture. Agroforest Syst, 61(1-3): 65-78. DOI: https://doi.org/10.1023/B:AGFO.0000028990.31801.62.

Brown Jr. K.S. \& Freitas A.L. 2002. Butterfly Communities of Urban Forest Fragments in Campinas, São Paulo, Brazil: Structure, Instability, Environmental Correlates, and Conservation. Journal of Insect Conservation, 6(4): 217-231.

DOI: https://doi.org/10.1023/A:1024462523826.

Brown Jr. K.S. 1972. Maximizing daily butterfly counts. Journal of the Lepidopterists' Society, 26(3): 183-196. http://images.peabody.yale.edu/lepsoc/jls/1970s/1972/19 72-26(3)183-Brown.pdf.

Brown Jr. K.S. 1978. Heterogeneidae: fator fundamental na teoria e prática de conservação de ambientes tropicais. Encontro Nacional sobre Preservação da Fauna e Recursos Faunísticos, Brasília 1977. 175-183. Brasília, IBDF.

Brown Jr. K.S. 1979. Ecologia Geográfica e Evolução nas Florestas Neotropicais. Campinas, SP: UNICAMP.

Brown Jr. K.S. 1982. Historical and ecological factors in the biogeography of aposematic neotropical Butterflies. Amer. $\quad$ Zool., $\quad$ 22(2): 453-471. https://www.jstor.org/stable/3882674.

Brown Jr. K.S. 1984. Species diversity and abundance in Jaru, Rondônia (Brazil). News Lepid. Soc., 26(3): 45-51. http://images.peabody.yale.edu/lepsoc/nls/1980s/1984/1 984_v26_n3.pdf.
Brown Jr. K.S. 1987a. Biogeography and evolution of Neotropical butterflies. In: Whitmore T.C. \& Prance G.T. (eds) Biogeography and Quaternary History in Tropical America. 66-104. Clarendon Press. Oxford.

Brown Jr. K.S. 1987b. Conclusions, synthesis, and alternative hypotheses. In: Whitmore T.C. \& Prance G.T. (eds) Biogeography and Quaternary History in Tropical America. 175-196. Clarendon Press. Oxford.

Brown Jr. K.S. 1988. Species diversity. In: Myers A.A. \& Giller P.S. (eds) Analytical Biogeography. 57-89. Chapman and Hall. London.

Brown K.S. 1997. Diversity, disturbance, and sustainable use of Neotropical forests: insects as indicators for conservation monitoring. Journal of Insect Conservation, 1(1): 25-42. https://doi.org/10.1023/A:1018422807610.

Butler R. 2017. Amazon insects. Mongabay. https://rainforests.mongabay.com/amazon/insects.html.

CADMA. 1992. Amazonia sin Mitos. Comisión Amazónica de Desarrollo y Medio Ambiente. UNDP/IADB. New York.

Cardoso P., Erwin T.L., Borges P.A.V. \& Newc T.R. 2011. The seven impediments in invertebrate conservation and how to overcome them. Biological Conservation, 144(11): 2647-2655. https://doi.org/10.1016/j.biocon.2011.07.024.

Castello L., McGrath D.G., Hess L.L., Coe M.T., Lefebvre P.A., Petry P., Macedo M.N., Renó V.F. \& Arantes C.C. 2012. The vulnerability of Amazon freshwater ecosystems. Conservation Letters, 6(4): 217-229. DOI: 10.1111/conl.12008.

Chapman A.D. 2009. Numbers of living species in Australia and the world. Report for the Australian Biological Resources Study. Canberra, Australia. https://www.environment.gov.au/system/files/pages/2ee 3f4a1-f130-465b-9c7a-79373680a067/files/nlsaw-2ndcomplete.pdf.

Clark A. \& May R.M. 2002. Taxonomic Bias in Conservation Research. Science, 297(5579): 191-192. DOI: $\quad 10.1126 /$ science.297.5579.191b. https://science.sciencemag.org/content/297/5579/191.2.

Collins N.M. \& Thomas J.A. (eds.). 1991. The Conservation of Insects and Their Habitats. Royal Entomological Society of London. Academic Press Ltd. DOI: https://doi.org/10.1002/rrr.3450070310.

Couceiro S.R.M., Hamada N., Luz S.L.B., Forsberg B.R. \& Pimentel T.P. 2007. Deforestation and sewage effects on aquatic macroinvertebrates in urban streams in Manaus, Amazonas, Brazil. Hydrobiologia, 575(1): 271-284. https://doi.org/10.1007/s10750-006-0373-z.

Dourojeanni M.J. \& Pádua M.T. 2007. Biodiversidade: A hora decisiva. Editora UFPR. Curitiba.

Dourojeanni M.J. \& Pádua M.T. 2013. Arcas à Deriva. Technical Books. Rio de Janeiro.

Dourojeanni M.J. \& Ponce C. 1978. Los Parques Nacionales del Perú. Instituto de la Caza Fotógrafica (INCAFO). Colección "La Naturaleza en Iberoamérica". Madrid, España.

Dourojeanni M.J. \& Tovar A. 1972. Notas sobre el ecosistema y la conservación de la Cueva de las Lechuzas (Parque Nacional de Tingo María, Perú). Revista Forestal del Perú, 5(1-2): 28-45.

Dourojeanni M.J. 1968. Estado actual de la conservación de la flora y la fauna en el Perú. Ciencia Interamericana, 9 (1-6): 1-12. 
Dourojeanni M.J. 1990. Entomology and biodiversity conservation in Latin America. American Entomologist, 36(2): 88-93.

Dourojeanni M.J. 2011. Amazonia Probable y Deseable. Ensayo sobre el presente y futuro de la Amazonía. Fondo Editorial de la Universidad Inca Garcilaso de la Vega. Lima.

Dourojeanni M.J. 2018a. Áreas Naturales Protegidas del Perú: El Comienzo. Grijley, Lima.

Dourojeanni M.J. 2018b. Áreas naturales protegidas e investigación científica en el Perú. Revista Forestal del Perú, 33(2): 91-101. DOI: http://dx.doi.org/10.21704/rfp.v33i2.1223.

Dourojeanni M.J. 2019. Conservation in the Amazon: Evolution and Situation. Environmental Science, Online Publication Date: Apr 2019. DOI: 10.1093/acrefore/9780199389414.013.41. Oxford Research Encyclopedia.

Dressler R.L 1985. Euglossine bees (Hymenoptera: Apidae) of the Tambopata Reserved Zone, Madre de Diós, Peru. Rev. Peruana Entom., 27: 75-9.

Emmel T.C. \& Austin G.T. 1990. The tropical rain forest butterfly fauna of Rondônia, Brazil: species diversity and conservation. Trop. Lepid., 1(1): 1-12.

Erwin T.L. 1982. Tropical forests: Their richness in Coleoptera and other Arthropod species. Coleopt. Bull. 36(1): 74-75

Erwin T.L. 1983a. Tropical forest canopies, the last biotic frontier. Bull. Entomol. Soc. Am., 29(1): 14-19.

Erwin T.L. 1983b. Beetles and other Arthropods of the tropical forest canopies at Manaus, Brazil, sampled with insecticidal fogging techniques. In: Sutton S.L., Whitmore T.C., \& Chadwick A.C. (eds) Tropical Rain Forests: Ecology and Management. 59-75. Blackwell Scientific Publications. Oxford.

Erwin T.L. 1985. Tambopata Reserved Zone, Madre de Dios, Peru: History and description of the Reserve. Rev. Peru. Entomol., 27: 1-8.

Erwin T.L. 1991a. An evolutionary basis for conservation strategies. Science, 253(5021): 750-752. DOI: 10.1126/science. 253.5021 .750 .

Erwin T.L. 1991b. Natural history of the carabid beetles at the BIOLAT biological station, Rio Manu, Pakitza, Peru. Rev. Peruana Entom., 33: 1-85.

Esquivel-Muelbert A., Baker T.R., Dexter K.G., Lewis S.L., Brienen R.J.W., Feldpausch T.R., et al. 2018. Compositional response of Amazon forests to climate change. Global Change Biology, 25(1): 35-56. DOI: $10.1111 / \mathrm{gcb} .14413$.

Fajardo J., Lessmann J., Bonaccorso E., Devenish C. \& Muñoz J. 2014. Combined use of systematic conservation planning, species distribution modelling, and connectivity analysis reveals severe conservation gaps in a megadiverse country (Peru). PLOS ONE, 9(12): e114367.

DOI: https://doi.org/10.1371/journal.pone.0114367.

Feeley K.J. \& Rehm E.M. 2012. Amazon's vulnerability to climate change heightened by deforestation and manmade dispersal barriers. Global Change Biology, 18(12): 3606-3614.
Fidelis L., Nessimian J.L. \& Hamada N. 2008. Distribuição espacial de insetos aquáticos em igarapés de pequena ordem na Amazônia Central. Acta Amazonica, 38(1): 127-134. $\quad$ http://dx.doi.org/10.1590/S004459672008000100014.

Fisher E.M. 1985. A preliminary list of the robberflies (Diptera: Asilidae) of the Tambopata Reserved Zone, Madre de Dios, Peru. Rev. Peruana Entom., 27: 25-36.

Foley J.A., Asner G.P., Costa M.H., Coe M.T., DeFries R., Gibbs H.K., Howard E.A., Olson S., Patz J., Ramankutty N. \& Snyder P. 2007. Amazonia revealed: forest degradation and loss of ecosystem goods and services in the Amazon Basin. Ecol Environ, 5(1): 25-32. The Ecological Society of America.

Foster R.B., Parker III T.A., Gentry A.H., Emmons L., Chicchón A., Schulenberg T., Rodríguez L., Lamas G., Ortega H., Icochea J. Wust W., Romo M., Castillo J.A., Phillips O., Reynel C., Kratter A., Donahue P.K. \& Barkley L.J. 1994. Tambopata-Candamo Reserved Zone. The Tambopata-Candamo Reserved Zone of southeastern Perú: A Biological Assessment.

Finer M. \& Mamani N. 2019. Hotspots de deforestación del 2018 en la amazonía peruana MAAP \#98. Monitoring of the Andean Amazon Project (MAAP). https://maaproject.org/2019/hotspot-peru-2018/.

Fox R., Harrower C.A, Bell J.R., Shortail C., Middlebrook I. \& Wilson R.J. 2018. Insect population trends and the IUCN Red List process. Journal of Insect Conservation, 23(2): 269-278. DOI: https://doi.org/10.1007/s10841018-0117-1.

Freitas B.M., Imperatriz-Fonseca V.L., Medina L.M., Kleinert A. de M.P., Galetto L., Nates-Parra G. \& Quezada-Euán J.G. 2009. Diversity, threats and conservation of native bees in the Neotropics. Apidologie, 40(3): 332-346. DOI: 10.1051/apido/2009012.

Garcia-Villacorta R., Dexter K.G. \& Pennington T. 2016. Amazonian White-Sand Forests Show Strong Floristic Links with Surrounding Oligotrophic Habitats and the Guiana Shield. Biotropica, 48(1): 47-57. DOI: 10.1111/btp.12302.

Gibbs G.W. 1978. The Role of the amateur entomologist in New Zealand. New Zealand Entomologist, 6(4): 328-334. DOI: https://doi.org/10.1080/00779962.1978.9722282.

Gibson L., Lee T.M., Koh L.P., Brook B.W., Gardner T.A., Barlow J., Peres C.A., Bradshaw C.J.A., Laurance W.F., Lovejoy T.E. \& Sodhi N.S. 2011. Primary forests are irreplaceable for sustaining tropical biodiversity. Nature, 478: 378-381. DOI: https://doi.org/10.1038/nature10425.

Grimwood I.R. 1968. Notes on the distribution and status of some Peruvian mammals 1968. American Committee for International Wild Life Protection (ACIWLP) (Now, AMERICAN COMMITTEE FOR INTERNATIONAL CONSERVATION, INC.). Special Publication Monographic Series N ${ }^{\circ} 21$. Bronx, N.Y.

Haddad N.M., Brudvig L.A., Clobert J., Davies K.F., Gonzalez A., Holt R.D., et al. 2015. Habitat fragmentation and its lasting impact on Earth's ecosystems. Science Advances, 1(2): e1500052. DOI: 10.1126/sciadv. 1500052 . 
Hallmann C.A., Sorg M., Jongejans E., Siepel H., Hofland N., Schwan H., Stenmans W., Müller A., Sumser H., Hörren T., Goulson D. \& de Kroon H. 2017. More than 75 percent decline over 27 years in total flying insect biomass in protected areas. PLOS ONE, 12(10): e0185809.

DOI: https://doi.org/10.1371/journal.pone.0185809.

Handunnetti D. 2019. An urban 'butterfly experience' in Sri Lanka. Mongabay, News of 15 May 2019. https://news.mongabay.com/2019/05/an-urban-butterflyexperience-in-srilanka/?n3wsletter\&utm_source=Mongabay.

Hebert P.D.N., Cywinska A., Ball S.L \& deWard J.R. 2003. Biological identification through DNA barcodes. Proceedings B (Biological Science), 270(1512): 313-321. The Royal Society of London. DOI: 10.1098/RSPB.2002.2218.

Hernández-Manrique O.L., Numa C., Verdú J., Galante E. \& Lobo J.M. 2012. Current protected sites do not allow the representation of endangered invertebrates: the Spanish case. Insect Conservation and Diversity, 5(6): 414-421. DOI: $10.1111 / \mathrm{j} .1752-4598.2011 .00175 . \mathrm{x}$.

Homma A.K.O. 2005. Biopirataria na Amazônia: como reduzir os riscos? Amazônia: Ciência e Desenvolvimento, 1(1): 47-60. Banco da Amazônia. Belém. https://www.bancoamazonia.com.br/index.php/compone nt/edocman/revista-amazonia-ciencia-edesenvolvimento-edicao-01/viewdocument?Itemid=. https://ainfo.cnptia.embrapa.br/digital/bitstream/item/30 451/1/BiopiratariaAmazonia.pdf.

IBAMA (Instituto Brasileiro do Meio Ambiente e dos Recursos Naturais Renováveis). 1991. Biological Priorities for Conservation in Amazonia. Workshop 90: Ações prioritárias para a conservação da biodiversidade da Amazônia. Ministério do Meio Ambiente, Brasília.

IBDF (Instituto Brasileiro de Desenvolvimento Florestal). 1982. Plano do Sistema de Unidades de Conservação do Brasil. Brasilia.

INPE (Instituto Nacional de Pesquisas Espaciais, BR). 2019. A estimativa da taxa de desmatamento por corte raso para a Amazônia Legal em 2019 é de 9.762 km². Instituto de Pesquisas Espaciais. Notícia, Nov 18, 2019. http://www.inpe.br/noticias/noticia.php?Cod_Noticia=5 294.

IPBES (Intergovernmental Science-Policy Platform on Biodiversity and Ecosystem Services). 2019. Summary for policymakers of the global assessment report on biodiversity and ecosystem services. Advance United $\begin{array}{llll}\text { Nations. } & 6 & \text { May } & 2019 .\end{array}$ https://ipbes.net/sites/default/files/downloads/spm_unedi ted_advance_for_posting_htn.pdf.

ISI (Initiative for Scottish Invertebrates). 2009. A strategy for Scottish invertebrate conservation. Ensuring important habitats, sites and endangered species are conserved. https://cdn.buglife.org.uk/2019/08/A-strategy-forScottish-invertebrate-conservation 0 .pdf.

IUCN (International Union for Conservation of Nature and Natural Resources). 2018. The IUCN Red List of Threatened Species. Version 2019-2. $<$ https://www.iucnredlist.org>.

https://www.iucnredlist.org/search?taxonomies $=100052$ \&search Type $=$ species
Jakovac C.C., Pena-Claros M., Kuyper Th.W. \& Bongers F. 2015. Loss of secondary-forest resilience by land-use intensification in the Amazon. Journal of Ecology, 103(1): 67-77. DOI: https://doi.org/10.1111/13652745.12298 .

Kress W.J., Heyer W.R., Acevedo P., Coddington J., Cole D., Erwin T.L., Megger B.J., Pogue M., Thorington R.W., Varis R.P., Weitzman M.J. \& Weitzman S.H. 1998. Amazonian biodiversity: assessing conservation priorities with taxonomic data. Biodiversity and Conservation, 7(12): 1577-1587. DOI: https://doi.org/10.1023/A:1008889803319.

Lamarre G.A., Decaens T., Rougerie R., Barbut J., Dewaard J.R., Hebert P.D.N., Herbin D., Laguerre M., Thiaucourtand P. \& Martins M.B. 2016. An integrative taxonomy approach unveils unknown and threatened moth species in Amazonian rainforest fragments. Insect Conservation and Diversity, 9(5): 475-479. DOI: 10.1111/icad.12187.

Lamas G. 1979. Algunas reflexiones y sugerencias sobre la creación de parques nacionales en el Perú. Ciencias, 71(1): 101-114.

Lamas G. 1985. Los Papilionoidea (Lepidoptera) de la Zona Reservada de Tambopata, Madre de Dios, Perú. I: Papilionidae, Pieridae y Nymphalidae (en parte). Rev. Peruana Entom., 27 : 59-73. https://cliniquevetodax.com/Eueides/LAMAS\%20\%20Papillons\%20de\%201a\%20r\%C3\%A9serve\%20Ta mbopata.pdf.

Laurance W.F. \& Vasconcelos H.L. 2009. Deforestation and forest fragmentation in the amazon. In: Tropical Biology and Conservation Management - Vol. II. 23-29. Encyclopedia of Life Support Systems (EOLSS). https://www.eolss.net/Sample-Chapters/C20/E6-142-HI03.pdf.

Laurance W.F., Camargo J.L.C., Luizao R.C.C., Laurance S.G., Pimm S.L., Bruna E.M., Stouffer P.C., Williamson G.B., Benitez-Malvido J., Vasconcelos H.L., Van Houtan K.S., Zartman C.E., Boyle S.A., Didham R.K., Andrade A. \& Lovejoy T.E. 2011. The fate of Amazonian forest fragments: A 32-year investigation. Biological Conservation, 144(1): 56-67. DOI: 10.1016/j.biocon.2010.09.021.

Le Page M. 2019. Huge global extinction risk for insects could be worse than we thought. NewScientist Environment, News of 11 February 2019. https://www.newscientist.com/article/2193494-hugeglobal-extinction-risk-for-insects-could-be-worse-thanwe-thought/.

Lewinsohn T.M. \& Prado P.I. 2005. How Many Species Are There in Brazil? Conservation Biology, 19(3): 619-624.

Liebhold A. \& Bentz B. 2011. Insect Disturbance and Climate Change. U.S. Department of Agriculture, Forest Service, Climate Change Resource Center. www.fs.usda.gov/ccrc/topics/insect-disturbance/insectdisturbance.

Lister B.C. \& Garcia A. 2018. Climate-driven declines in arthropod abundance restructure a rainforest food web. PNAS (Proceedings of the National Academy of Sciences USA), 115(44): E10397-E10406. https://doi.org/10.1073/pnas.1722477115. 
Longino J.T. 2000. Review: Paint-by-Numbers Ecology. Reviewed Work: "Nature in Focus: Rapid Ecological Assessment" by Roger Sayre, Ellen Roca, Gina Sedaghatkish, Bruce Young, Shirley Keel, Roberto Roca, Stuart Sheppard. 1999. Nature in Focus: Rapid Ecological Assessment. Ecology, 81(9): 2646-2647. DOI: $10.2307 / 177484$.

Lovejoy T.E. \& Nobre C. 2018. Amazon Tipping Point. Science Advances, 4(2): eaat2340. DOI: 10.1126/sciadv.aat2340.

Lovejoy T.M., Bierregaard R.O., Rylands A.B., Malcolm J.R., Quintela C.E., Harper J.H., Brown K.S. Jr, Powell A.H., Powell G.V.N., Schubart H.O.R. \& Hays M.B. 1986. Edge and other effects of isolation on Amazon forest fragments. In: Soulé M.E. (ed.) Conservation Biology: The Science of Scarcity and Diversity. 257-285. Sunderland, Mass.: Sinauer.

Malhi Y., Roberts J.T. , Betts R.A., Killeen T.J., Li W. \& Nobre C.A. 2008. Climate Change, Deforestation, and the Fate of the Amazon. Science, 319(5860): 169-172. DOI: 10.1126/science.1146961.

Marques E.Q., Marimon-Junior B., Marimon B.S., Matricardi E.A.T., Mews H.A. \& Coll G.R. 2019. Redefining the Cerrado-Amazonia transition: implications for conservation. Biodiversity and Conservation, First $\begin{array}{llll}\text { Online: } & 25 & \text { February } & 2019 .\end{array}$ https://doi.org/10.1007/s10531-019-01720-z.

Martins M.B. 1989. Invasão de fragmentos florestais por espécies oportunistas de Drosophila (Diptera, Drosophilidae). Acta Amazonica, 19: 265-271.

McGeoch M.A., Sithole H., Samways M.J., Simaika J.P., Pryke J.S., Picker M., Uys Ch., Armstrong A.J., Dippenaar-Schoeman A.S., Engelbrecht I.A., Braschler B., Hamer M. 2011. Conservation and monitoring of invertebrates in terrestrial protected areas. Koedoe, 53(2): 1000. Pretoria Jan. 2011. DOI: 10.4102/koedoe.v53i2.1000.

Miller S.E. 2007. DNA barcoding and the renaissance of taxonomy. PNAS (Proceedings of the National Academy of Sciences USA), 104(12): 4775-4776. DOI: 10.1073/pnas.0700466104.

Miranda E.A., Carvalho A.F., A.F. de Jesus Gomes-Miranda, J.C. Ribeiro de Souza \& M.A. Costa. 2019. Priority areas for conservation of orchid bees (Apidae, Euglossini) in the Atlantic Forest. J. Insect Conserv., 23(3): 613-621. https://doi.org/10.1007/s10841-019-00155-9.

Morrone J.J. 1994. On the identifcation of areas of endemism. Syst. Biol., 43: 438-441.

Mittermeier R.A., Mittermeier C. \& Robles P. 1997. Megadiversity: Earth's biologically wealthiest nations. Cemex, México.

National Research Council. 1982. Ecological aspects of development in the humid tropics. Committee on Selected Biological Problems in the Humid Tropics, Division of Biological Sciences, Assembly of Life Sciences, National Research Council / U.S. Washington, D.C. National Academy Press. https://www.nap.edu/catalog/19533/ecological-aspectsof-development-in-the-humid-tropics.

Nelson B.W., Ferreira C.A.C., da Silva M.F. \& Kawasaki M.L. 1990. Endemism centres, refugia and botanical collection density in Brazilian Amazonia. Nature, 345: 714-716.
Nessimian J.L., Venticinque E.M., Zuanon J., De Marco Jr. P., Gordo M., Fidelis L., D’arc Batista J., Juen L. 2008. Land use, habitat integrity, and aquatic insect assemblages in Central Amazonian streams. Hydrobiologia, 614: 117-131. DOI: 10.1007/s10750008-9441-x.

Oliveira U., Pereira Paglia A., Brescovit A.D., de Carvalho C.J.B., Paiva Silva D., Rezende D.T., Sá Fortes Leite F., Aguiar Nogueira Batista J., et al. 2017a. The strong influence of collection bias on biodiversity knowledge shortfalls of Brazilian terrestrial biodiversity. Diversity and Distributions, 22(12): 1232-1244. https://doi.org/10.1111/ddi.12489.

Oliveira U., Silveira Soares-Filho B., Pereira Paglia A., Brescovit A.D., de Carvalho C.J.B., Paiva Silva D., Rezende D.T., Sá Fortes Leite F., Aguiar Nogueira Batista J., Peixoto Pena Barbosa J.P., Stehmann J.R., Ascher J.S., Ferreira de Vasconcelos M., De Marco P., Löwenberg-Neto P., Gianluppi Ferro V. \& Santos A.J. 2017b. Biodiversity conservation gaps in the Brazilian protected areas. Scientific Reports, 7: 9141. DOI: 10.1038/s41598-017-08707-2.

Pádua M.T. \& Coimbra A.F. 1979. Os Parques Nacionais do Brasil. Incafo, Madrid.

Parra G.N. \& González V.H. 2000. The Colombian wild bees: Why and how to preserve them. Acta biol. Colomb., 5(1): 5-37.

Paulson D.R. 1985. Odonata of the Tambopata Reserved Zone, Madre de Dios, Peru. Rev. Peruana Entom., 27: 914.

Pearson D.L. 1985. The Tiger Beetles (Coleoptera: Cicindellidae) of the Tambopata Reserved Zone, Madre de Dios, Peru. Rev. Peruana Entom., 27: 15-24.

Pearson D.L. 1992. Tiger beetles as indicators for biodiversity patterns in Amazonia. Natl. Geogr. Soc. Res. \& Explor., 8: 116-117.

Pearson D.L. 1994. Selecting indicator taxa for the quantitative assessment of biodiversity. Philosophical Transactions B, 345(1311): 75-79. London.

Perry J., Lojk B., Quinones L.G., Van Damme P., Houška J. \& Fernandez E. 2016. How natural Forest Conversion Affects Insect Biodiversity in the Peruvian Amazon: Can Agroforestry Help? MDPI. Forests, 7(4): 82. DOI: https://doi.org/10.3390/f7040082. https://www.mdpi.com/1999-4907/7/4/82.

Pitman N., Smith R.C., Vriesendorp C., Moskovits D., Piana R., Knell G. \& Wachter T. (eds.). 2004. Perú: Ampiyacu, Apayacu, Yaguas, Medio Putumayo. Rapid Biological Inventories Report 12. The Field Museum. Chicago, Illinois, USA.

Powell A.H. \& Powell G.V.N. 1987. Population Dynamics of Male Euglossine Bees in Amazonian Forest Fragments. Biotropica, 19(2): 176-179. DOI: 10.2307/2388742.

Prado S.G., Ng H.T. \& Florez J.A. 2017. Sampling bees in tropical forests and agroecosystems: a review. Journal of Insect Conservation, 21(5-6): 753-770.

Prance G.T. 1982. Forest refuges: evidence from woody angiosperms. In: Prance G.T. (ed.) Biological Diversification in the Tropics. 137-157. Columbia Univ. Press. New York.

Rafael J.A., Melo G.A.R., Carvalho C.J.B., Casari A.S. \& Constantino R. (eds.) 2012. Insetos do Brasil Diversidade e taxonomia. Holos Editora. Ribeirão Preto. 
RAISG (Red Amazónica de Información Socioambiental Georreferenciada). 2019. Amazônia 2019 - Áreas Protegidas - Territorios Indígenas en la Amazonía (mapa). En: Red Amazónica de Información Socioambiental Georreferenciada. https://www.amazoniasocioambiental.org/es/publicacion /amazonia-2019-areas-protegidas-y-territoriosindigenas/.

Robbins R.K., Lamas G., Mielke O.H.H., Harvey D.J. \& Casagrande M.M. 1997. Taxonomic composition and ecological structure of the species-rich butterfly community at Pakitza, Parque Nacional del Manu, Peru. In: Wilson D. \& Sandoval A. (eds.) Manu: La Biodiversidad del Sureste del Perú - Manu: The Biodiversity of Southeastern Peru. Smithsonian Institution, National Museum of Natural History (Smithsonian Institution) and Editorial Horizonte. 217252.

Rosenberg D.K., Noon B.R. \& Meslow E.Ch. 1997. Biological Corridors: Form, Function, and Efficacy. BioScience, 47(10): 677-687. DOI https://doi.org/10.2307/1313208.

Roubik D.W. \& Hanson P.H. 2004. Abejas de Orquídeas de la América Tropical: Biología y Guía de Camp. INBIO. San José, Costa Rica.

Samways M.J. 2005. Insect Diversity Conservation. Cambridge.

Sánchez-Bayo F. \& Wyckhuys K.A.G. 2019. Worldwide decline of the entomofauna: A review of its drivers. Biological Conservation, 232: 8-27. DOI: 10.1016/j.biocon.2019.01.020.

Schwarz H.F. 1932. The genus Melipona: the type genus of the Meliponidae or stingless bees. Bulletin of the American Museum of Natural History, 63(4): 1883-1960.

Schulenberg T.S. \& Awbrey K. (eds.). 1997. The Cordillera del Condor of Ecuador and Peru: A Biological Assessment. RAP Working Papers, 7: 1-231.

SERFOR. 2018. Libro Rojo de la Fauna Silvestre Amenazada del Perú. $1^{\text {a }}$ Ed. Servicio Nacional Forestal y de Fauna Silvestre. Lima, Perú.

Silva Freitas da J., A. Mathis, Cordeiro Farias Filho M., Oyama Homma A.K. \& Costa Correia Silva D. 2017. Reservas extrativistas na Amazônia: Modelo de conservação ambiental e desenvolvimento social? GEOgraphia, 19(40): 150-160. Universidade Federal Fluminense. Niterói. DOI: https://doi.org/10.22409/GEOgraphia2017.v19i40.a1380 $\underline{6}$. https://ainfo.cnptia.embrapa.br/digital/bitstream/item/16 4792/1/1020-4864-1-PB.pdf.
Simaika J.P. \& Samways M.J. 2018. Insect conservation psychology. Journal of Insect Conservation, 22(3-4): 635-642. DOI: https://doi.org/10.1007/s10841-0180047-y.

Souza C. Jr. \& Roberts D. 2005. Mapping forest degradation in the Amazon region with Ikonos images. International Journal of Remote Sensing, 26: 425-429. DOI: https://doi.org/10.1080/0143116031000101620.

Tosi J. 1960. Zonas de Vida Natural en el Perú. IICA, Zona Andina Informe No 5. Lima.

Tovar A., Arnillas C.A., Eckhardt K., Regal F. \& Véliz C. 2009. Sierra del Divisor: Una Sierra al Este del Ucayali. Lima, Perú. CONCYTEC, Centro de Datos para la Conservación.

Vasconcelos H.L. \& Bruna E.M. 2012. Arthropod responses to the experimental isolation of Amazonian forest fragments. Zoologia (Curitiba), 29(6): 515-530. http://dx.doi.org/10.1590/S1984-46702012000600003.

Vicente R.E., Pires Prado L. \& Junqueira Izzo T. 2016. Amazon Rainforest Ant-Fauna of Parque Estadual do Cristalino: Understory and Ground-Dwelling Ants. Sociobiology, 63(3): 894-908. DOI: http://dx.doi.org/10.13102/sociobiology.v63i3.1043. http://periodicos.uefs.br/index.php/sociobiology/article/v iew/1043.

Vriesendorp C. (Ed.). 2006. Perú: Sierra del divisor. The Field Museum, Rapid Biological Inventories 16.

Wetterberg G.B., Pádua M.T. J., Castro C.S. de. \& Vasconcelos J.M.C. de. 1976. Uma análise de prioridades em conservação da natureza na Amazônia. PNUD/FAO/IBDF/BRA.45 IBDF, Brasília, DF. Série Técnica $\mathrm{N}^{\circ} 8$.

Wilkerson R.C. \& Fairchild G.B. 1985. A checklist and generic key to the Tabanidae (Diptera) of Peru with special reference to the Tambopata Reserved Zone, Madre de Diós. Rev. Peruana Entom., 27: 37-53.

Wilson E.O. 1987. The little things that run the world (the importance and conservation of invertebrates). Conserv. Biol., 1: 344-346.

Yang H.P., Ma C.-S., Wen H., Zhan Q.-B. \& Wang X.-L. 2015. A tool for developing an automatic insect identification system based on wing outlines. Scientific Reports, $\quad$ 5: $12786 . \quad$ DOI: https://doi.org/10.1038/srep12786.

\footnotetext{
${ }^{1}$ Ingeniero Agrónomo, Ingeniero Forestal, Doctor en Ciencias. Profesor Emérito de la Universidad Nacional Agraria La Molina. Lima / Perú.

Se terminó de redactar en julio de 2019.
} 